\title{
Inferior Vena Cava Distensibility in Patients with SAH, New Technology and Numbers, Better Care?
}

\author{
Clemens M. Schirmer
}

Published online: 11 May 2010

(C) Springer Science+Business Media, LLC 2010

\begin{abstract}
Sometimes I wonder whether today we take sufficient care to make a thorough physical examination before our patient starts off on the round of the laboratories, which have become so necessary that oftentimes we do not fully appreciate the value of our five senses in estimating the condition of the patient.-William Mayo, Collected papers of the Mayo Clinic, 1938
\end{abstract}

The use of bedside ultrasound examination by intensivists is a recent development, for the neurocritical care specialist this complements the longstanding experience neurosurgeons and neurologists have with transcranial Doppler examinations for vasospasm. Drs. Moretti and Pizzi report in this issue on their experience using bedside ultrasound examination to predict fluid responsiveness in patients with subarachnoid hemorrhage (SAH), using inferior vena cava distensibility (dIVC) as a parameter that discerns between fluid responders and non-responders. They enrolled 29 adult patients with SAH requiring advanced hemodynamic monitoring, measured the inferior vena cava diameter during a single mechanical breath and the dIVC was calculated. A dIVC value of $>16 \%$ yielded the best predictive value for volume response. There was a trend toward a lower incidence of delayed ischemic lesions in fluid responders and the dIVC was determined a reliable predictor of fluid responsiveness in patients with SAH [1].

The article might be criticized on multiple levels, one could ask for a larger number of patients or a longer follow-up but more importantly it accomplishes a number of goals for the interested reader-it introduces a new

C. M. Schirmer $(\bowtie)$

Department of Neurosurgery, Tufts Medical Center and Tufts University School of Medicine, Boston, MA, USA

e-mail: cschirmer@tuftsmedicalcenter.org technology application, or better, a new measurement obtainable with the same ultrasound machine that might be already available in the intensive care unit. It makes the case for the dIVC compared to other hemodynamic parameters that attempt to assess volume status in the critically ill patient and establishes its use in the clinical scenario of patients with SAH.

Substituting the subjective assessment of a patient's status by a clinician with objective measures uncouples the assessment from the experience of the clinician taking the measurement. On the other hand, it enables objective comparison and research. Technology may become used in its own right or as a substitute and not as an adjunct for good clinical care. The words of William Mayo above may be a reminder that remains current. A study published in the Lancet a few years ago demonstrated that the findings on physical examination by an attending physician were pivotal in the management of more than a quarter of all medical patients [2]. This should give us more reason for pause and appropriate evaluation of new technology before widespread use complicates this evaluation.

What is the optimal parameter to assess volume status? The current debate in the critical care literature on this issues is lively and appears by no means settled: Marik and colleagues, using statistical analysis and data from controlled studies, show that isolated central venous pressure (CVP) measurements are a poor guide to the assessment of volume status and fluid responsiveness [3]. They argue that with certain exceptions, where right ventricular function rather than volume status is assessed, "CVP should no longer be routinely measured in the ICU, operating room, or emergency department" [3]. Singh et al. disagree and counter that it is the intuitive simplicity of CVP that contributed to its widespread use because it comes with convenience and there are no controlled studies that prove 
definite disadvantage related to the method used for fluid management [4]. The current study tries to make the case that dIVC is the optimal guide for volume status for the patient with SAH and may be convincing, however, given the debate about the underlying assumption this conclusion should be at least be interpreted with caution.

Why do we need to know about the fluid responsiveness of patients with SAH? Lacking a better alternative the gold standard aiming for the prevention and treatment of vasospasm has been the combination of hypertension, hypervolemia, and hemodilution, also known as triple- $\mathrm{H}$ therapy. The scientific basis for this treatment was recently questioned a review by Dankbaar et al. [5]. The different triple-H components aim to influence perfusion pressure and blood viscosity in order to increase cerebral blood flow (CBF) but it is unclear whether strategies altering hemodilution and hypervolemia actually achieve these effects, nor could they confirm that the combination of all three components, which should theoretically result in the largest CBF increase delivers any benefit [5].

The authors of the current study should be commended for taking the first step toward pointing our interest in the management of patients with SAH in a slightly new direction where a simple ultrasound examination determines the fluid responsiveness. Further studies might be necessary to establish important questions including validity and how the use of the dIVC to guide goal-directed fluid therapy in SAH patients translates into distinct clinical outcomes.

\section{References}

1. Moretti R, Pizzi B. Inferior vena cava distensibility as a predictor of fluid responsiveness in patients with subarachnoid hemorrhage. Neurocrit Care. 2010 [Epub ahead of print]. PubMed PMID: 20373051.

2. Reilly BM. Physical examination in the care of medical inpatients: an observational study. Lancet. 2003;362(9390):1100-5.

3. Marik PE, Baram M, Vahid B. Does central venous pressure predict fluid responsiveness? A systematic review of the literature and the tale of seven mares. Chest. 2008;134(1):172-8.

4. Singh NK, Sangwan G, White P. Central venous pressure as popular resuscitation surrogate: not totally unjustified. Chest. 2008; 134(6): 1352. author reply 1352-3.

5. Dankbaar JW, Slooter AJ, Rinkel GJ, Schaaf IC. Effect of different components of triple-H therapy on cerebral perfusion in patients with aneurysmal subarachnoid haemorrhage: a systematic review. Crit Care. 2010;14(1):R23. 